\title{
Numerical comparison of CBS and SGS as stabilization techniques for the incompressible Navier-Stokes equations
}

\author{
R. Codina ${ }^{1, *, \dagger}$, H. Coppola-Owen ${ }^{1, \ddagger}$, P. Nithiarasu ${ }^{2, \S}$ and C.-B. Liu ${ }^{2, \mathbb{I}}$ \\ ${ }^{1}$ Universitat Politècnica de Catalunya, Jordi Girona 1-3, 08034 Barcelona, Spain \\ ${ }^{2}$ Civil and Computational Engineering Centre, School of Engineering, University of Wales Swansea, \\ Swansea SA2 8PP, U.K.
}

\begin{abstract}
SUMMARY
In this work, we present numerical comparisons of some stabilization methods for the incompressible Navier-Stokes. The first is the characteristic-based split (CBS). It combines the characteristic Galerkin method to deal with convection-dominated flows with a classical splitting technique, which in some cases allows us to use equal velocity-pressure interpolations. The other two approaches are particular cases of the subgrid scale (SGS) method. The first, obtained after an algebraic approximation of the subgrid scales, is very similar to the popular Galerkin/least-squares (GLS) method, whereas in the second, the subscales are assumed to be orthogonal to the finite element space. It is shown that all these formulations display similar stabilization mechanisms, provided the stabilization parameter of the SGS methods is identified with the time step of the CBS approach. This paper provides the numerical experiments for the comparison of formulations made by Codina and Zienkiewicz in a previous article. Copyright (c) 2006 John Wiley \& Sons, Ltd.
\end{abstract}

KEY WORDS: incompressible flows; CBS; stabilization

\section{INTRODUCTION}

The Navier-Stokes equations for an incompressible fluid moving in a domain $\Omega$ during the time interval $] 0, T[$ can be written as

$$
\begin{array}{r}
\partial_{t} \mathbf{u}+\mathbf{u} \cdot \nabla \mathbf{u}-v \Delta \mathbf{u}+\nabla p=\mathbf{f} \\
\nabla \cdot \mathbf{u}=0
\end{array}
$$

\footnotetext{
*Correspondence to: R. Codina, Campus Nord UPC, Edifici C1, Jordi Girona 1-3, 08034 Barcelona, Spain.

†E-mail: ramon.codina@upc.edu

\#E-mail: howen@cimne.upc.es

${ }^{\S}$ E-mail: p.nithiarasu@swansea.ac.uk

IE-mail: 202827@swansea.ac.uk
} 
where $\mathbf{u}$ is the velocity, $p$ the pressure, $\mathbf{f}$ the vector of body forces and $v$ the kinematic viscosity. These equations have to be supplied with an initial condition of the form $\mathbf{u}=\mathbf{u}_{0}$ at $t=0$ and a boundary condition which, for simplicity, will be taken as $\mathbf{u}=\mathbf{0}$ on $\partial \Omega$.

Whichever the time discretization method of (1) is, the spatial discretization may suffer from two major numerical problems. The first is the velocity-pressure interpolation. The zero divergence restriction imposes a compatibility condition for this interpolation that, in particular, prevents the use of equal interpolation for the velocity and the pressure. Likewise, when convection dominates, spurious spatial oscillations may occur if the standard Galerkin formulation is used, similarly to what happens with centred finite difference schemes. This has to be understood as a numerical problem, and is independent of the complicated flow features (including turbulence) that appear when the Reynolds number is high. The parameter that plays a role in this case is the cell Reynolds number rather than the global one. See Reference [1] for background.

These two numerical problems have been treated with a variety of finite element formulations. The objective of this paper is precisely to present a numerical comparison for some of them, namely, the CBS (see References [2-4]) and two versions of the subgrid scale (SGS) method proposed in Reference [5], one similar to the Galerkin/least-squares (GLS) method (see References [6,7]) and fully described in Reference [8] and the other obtained after assuming that the subscales are orthogonal to the finite element space, and presented in Reference [9]. We call the first approach algqbraic SGS (ASGS) and the second orthogenal SGS stabilization (OSS). Both these SCS methods and CBS are consistent and, at least for both ASGS and OSS, known to converge optii accuracy depends on

The idea of the CBS scheme is to stabilize convection by using along the characteristics, and to rely on the stabilizing effec ting technique. On the other hand, the SGS methods provide control on the element residual of

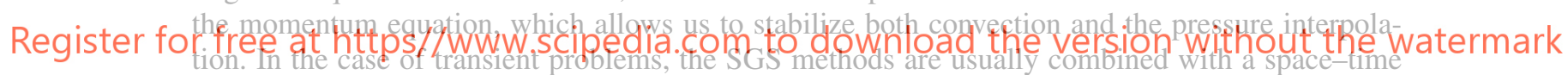
finite element formulation (see, e.g. Reference [10]), and this is what we will do here.

It is not the purpose of this paper to motivate the different methods. The theoretical comparison between CBS and ASGS was done in Reference [11] (there, the GLS method was taken as a reference instead of ASGS, but both are similar for our comparison purposes). This work can be understood as a complement to Reference [11], in the sense that it contains the numerical comparison that was not presented there. Therefore, the different formulations will be simply described and then the numerical examples presented. The CBS scheme used for the solution of problems presented in Section 3 makes use of a fully explicit artificial compressibility form described in References [12,13].

\section{CBS AND SGS METHODS}

\subsection{The characteristic-based splitting (CBS) method}

The CBS method is based on a discretization of the time derivative in the momentum equation along the characteristics and a classical pressure splitting. Let us see how to apply these two numerical ingredients. 
Consider a uniform partition of $[0, T]$ into time intervals of equal size $\delta t$. Suppose now that we have the solution at time $t_{n}=n \delta t$ and we want to compute it at time $t_{n+1}=t_{n}+\delta t$. Denoting by a superscript the time step level and treating the discretization along the characteristics explicitly, the time discretized problem is

$$
\begin{gathered}
\frac{1}{\delta t}\left(\mathbf{u}^{n+1}-\mathbf{u}^{n}\right)+\mathbf{u}^{n+1 / 2} \cdot \nabla \mathbf{u}^{n+1-\gamma}-v \Delta \mathbf{u}^{n+1 / 2}+\nabla p^{n+1 / 2}-\mathbf{f}^{n+1 / 2} \\
-(2 \gamma-1) \frac{\delta t}{2} \mathbf{u}^{n} \cdot \nabla\left(\mathbf{u}^{n} \cdot \nabla \mathbf{u}^{n}-v \Delta \mathbf{u}^{n}+\nabla p^{n}-\mathbf{f}^{n}\right)=\mathbf{0} \\
\nabla \cdot \mathbf{u}^{n+1}=0
\end{gathered}
$$

where $t^{n}+\gamma \delta t$ is the time at which the discretization along the characteristics is done, with $1 / 2<\gamma \leqslant 1$. This discretized problem is obtained by using the Crank-Nicolson discretization of the total time derivative. However, there is also the possibility of using an explicit scheme, treating only the pressure implicitly or using an artificial compressibility method to allow its explicit treatment, as explained in Reference [12]. This possibility is used in the numerical examples.

\section{We may interpret (3)-(4) as the time discretization of problem (1)-(2) plus the introduction}
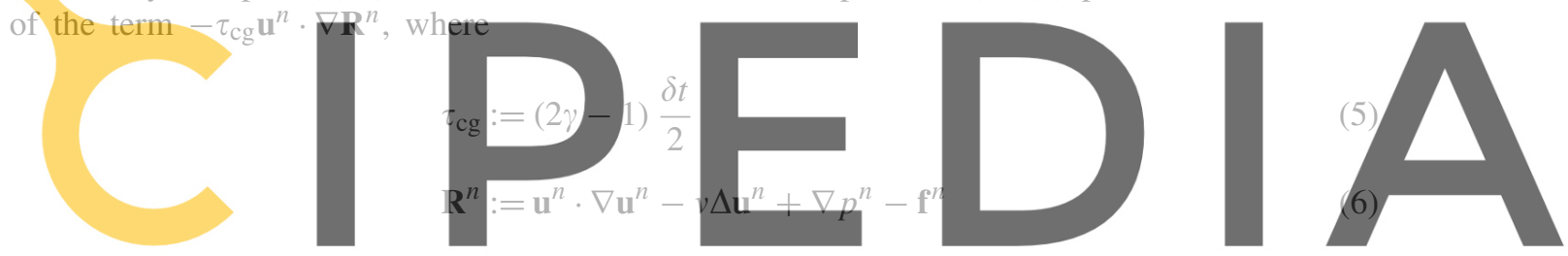

The next ingredient of the CBS algorithm is a fractional step method to segregate the pressure

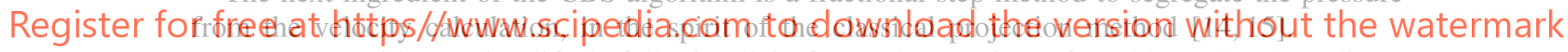

Let us consider a simplified, fully implicit first-order version of problem (3)-(4) split as follows:

$$
\begin{aligned}
\frac{1}{\delta t}\left(\hat{\mathbf{u}}^{n+1}-\mathbf{u}^{n}\right)+\hat{\mathbf{u}}^{n+1} \cdot \nabla \hat{\mathbf{u}}^{n+1}-v \Delta \hat{\mathbf{u}}^{n+1}+\gamma^{\prime} \nabla p^{n}-\mathbf{g}^{n+1} & =\mathbf{0} \\
\frac{1}{\delta t}\left(\mathbf{u}^{n+1}-\hat{\mathbf{u}}^{n+1}\right)+\nabla p^{n+1}-\gamma^{\prime} \nabla p^{n} & =\mathbf{0} \\
\nabla \cdot \mathbf{u}^{n+1} & =0
\end{aligned}
$$

where $\hat{\mathbf{u}}^{n+1}$ is an intermediate unknown, $\gamma^{\prime}$ a numerical parameter and $\mathbf{g}^{n+1}:=\mathbf{f}^{n+1}+$ $\tau_{\mathrm{cg}} \mathbf{u}^{n} \cdot \nabla \mathbf{R}^{n}$. We will proceed here very formally, assuming that the same boundary conditions can be applied for $\hat{\mathbf{u}}^{n+1}$ and the end-of-step velocity $\mathbf{u}^{n+1}$.

We will not explore here the stabilization mechanism identified in Reference [2], but rather the approach noted in Reference [16]. The difference between both points of view is that while in Reference [2] we analyse the problem for the end-of-step velocity, in Reference [16] the author points out the stabilized problem of which the intermediate velocity is solution. Here, we pursue this viewpoint. 
The splitting error introduced in (7)-(9) comes from the fact that the viscous and the convective terms are computed with $\hat{\mathbf{u}}^{n+1}$ instead of $\mathbf{u}^{n+1}$. This error is of second order if $\gamma^{\prime}=1$ and of first order otherwise.

It is interesting to eliminate the end-of-step velocity at time step $n, \mathbf{u}^{n}$, from (7) using (8) evaluated at time step $n$, and also $\mathbf{u}^{n+1}$ from (9) using (8) at time step $n+1$. This yields

$$
\begin{aligned}
\frac{1}{\delta t}\left(\hat{\mathbf{u}}^{n+1}-\hat{\mathbf{u}}^{n}\right)+\hat{\mathbf{u}}^{n+1} \cdot \nabla \hat{\mathbf{u}}^{n+1}-v \Delta \hat{\mathbf{u}}^{n+1}+\left(1+\gamma^{\prime}\right) \nabla p^{n}-\gamma^{\prime} \nabla p^{n-1}-\hat{\mathbf{g}}^{n+1} & =\mathbf{0} \\
\nabla \cdot \hat{\mathbf{u}}^{n+1}-\delta t\left(\Delta p^{n+1}-\gamma^{\prime} \Delta p^{n}\right) & =0
\end{aligned}
$$

where the stabilizing term in $\hat{\mathbf{g}}$ is evaluated with $\hat{\mathbf{u}}^{n}$ instead of $\mathbf{u}^{n}$, which implies an approximation of order $\theta\left(\delta t^{2}\right)$. For $n=0$ we consider $p^{-1}=p^{0}$, which is computed from the Poisson equation obtained by taking the divergence of the original momentum equation (1) and evaluating it at $t=0$.

It is important to note that problem (10)-(11) is unconditionally stable, even though the pressure is treated explicitly (and thus its calculation can be uncoupled from the velocity). This follows directly from the stability of the original problem (7)-(9), proven for example in Reference [17].

Usually, for $\theta \in[0,1]$ one defines $p^{n+\theta}:=\theta p^{n+1}+(1-\theta) p^{n}$. However, we could let $\theta \in[1,2]$

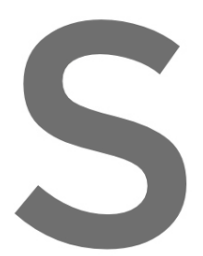
and consider $p$ pressure (rather than

Let us define the
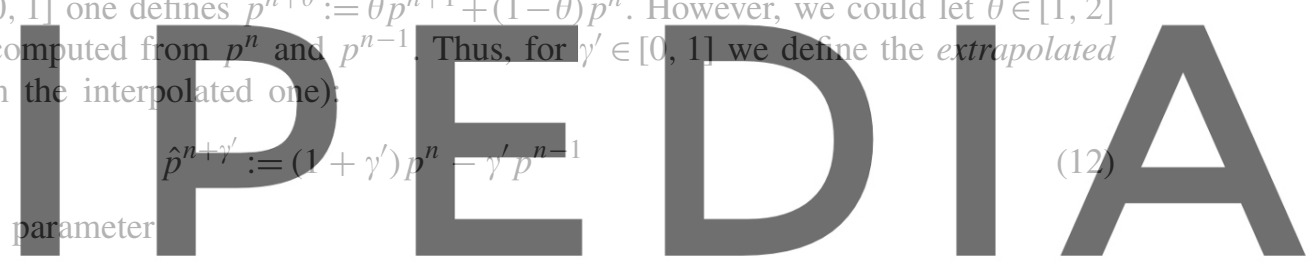

$$
\tau_{s}:=\delta t\left(1-\gamma^{\prime}\right)
$$

(13)

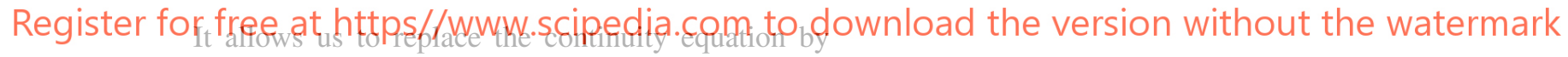

$$
\nabla \cdot \hat{\mathbf{u}}^{n+1}-\tau_{s} \Delta p^{n+1}=0
$$

which differs from (11) by the term $\delta t \gamma^{\prime}\left(\Delta p^{n+1}-\Delta p^{n}\right)$, which is of order $\mathcal{O}\left(\delta t^{2}\right)$.

Let $V_{h}$ be the velocity finite element space, incorporating the Dirichlet boundary conditions, and $Q_{h}$ the pressure space. As it is usual in the case of fractional step methods, the natural pressure boundary condition is zero normal derivative, which is a consequence of (8) and the fact that $\mathbf{u}$ and $\hat{\mathbf{u}}$ satisfy the same boundary conditions.

Integrating by parts the term coming from the discretization along the characteristics, it is found that the fully discrete form of problem (10)-(14) is: find $\hat{\mathbf{u}}_{h}^{n+1} \in V_{h}$ and $p_{h}^{n+1} \in Q_{h}$ such that

$$
\begin{aligned}
& \int_{\Omega} {\left[\frac{1}{\delta t}\left(\hat{\mathbf{u}}_{h}^{n+1}-\hat{\mathbf{u}}_{h}^{n}\right) \cdot \mathbf{v}_{h}+v \nabla \hat{\mathbf{u}}_{h}^{n+1}: \nabla \mathbf{v}_{h}+\left(\hat{\mathbf{u}}_{h}^{n+1} \cdot \nabla \hat{\mathbf{u}}_{h}^{n+1}\right) \cdot \mathbf{v}_{h}-\hat{p}_{h}^{n+\gamma^{\prime}} \nabla \cdot \mathbf{v}_{h}\right.} \\
&+\left.\tau_{\mathrm{cg}}\left(\hat{\mathbf{u}}_{h}^{n} \cdot \nabla \mathbf{v}_{h}\right) \cdot \hat{\mathbf{R}}_{h}^{n}-\mathbf{f}^{n+1} \cdot \mathbf{v}_{h}\right] \mathrm{d} \Omega=0 \\
& \int_{\Omega}\left[q_{h} \nabla \cdot \hat{\mathbf{u}}_{h}^{n+1}+\tau_{s} \nabla q_{h} \cdot \nabla p_{h}^{n+1}\right] \mathrm{d} \Omega=0
\end{aligned}
$$


for all test functions $\mathbf{v}_{h} \in V_{h}$ and $q_{h} \in Q_{h}$. The term $\hat{\mathbf{R}}_{h}^{n}$ is computed as indicated in (6) using $\hat{\mathbf{u}}_{h}^{n}$ instead of $\mathbf{u}^{n}$ and evaluating the second derivatives appearing in the viscous term element by element.

Problem (15)-(16) is the CBS finite element formulation that we compare in next section with the SGS methods. The stabilization mechanisms introduced by this formulation are now clear: a streamline diffusion introduced by the discretization along the characteristics stabilizes convection and the splitting introduces a pressure Laplacian, similarly to the stabilized methods analysed for example in Reference [18]. Note that in our case this stabilizing effect disappears if a second-order splitting is used (i.e. if $\gamma^{\prime}=1$ ).

\subsection{Two SGS methods}

Contrary to the CBS method, SGS formulations deal with convection and pressure stabilization using the same approach. The idea of SGS methods was proposed in Reference [5], although it is inherent in other numerical formulations. Our presentation differs from the one described in this reference, especially in the approximation of the SGS (it is an extension of the method presented in Reference [8] to the transient case).

If $\mathbf{u}_{h}$ and $p_{h}$ are the finite element unknowns of the problem, the key idea is to approximate $\mathbf{u} \approx \mathbf{u}_{h}+\widetilde{\mathbf{u}}$ and $p \approx p_{h}$, that is, the velocity is approximated by its finite element component

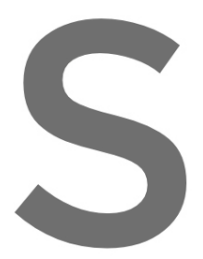
plus an additional terin

As in the case of $\mathbf{u}^{n+1} \approx \mathbf{u}_{*}^{n+1}:=\mathbf{u}_{h}^{n+1}$

the spatial interpolation finite element interpo

The important poi
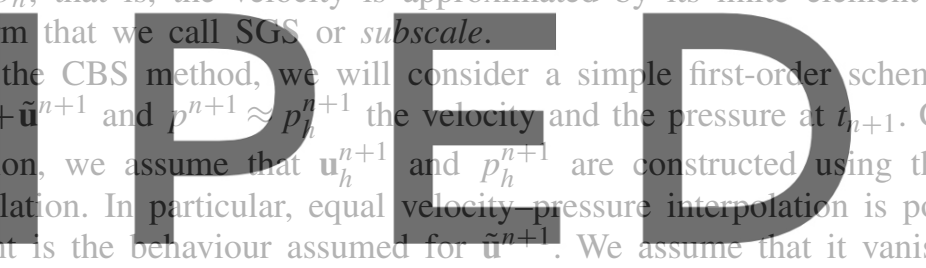

interelement boundaries, that is, it is a bubble-like function $[19,20]$. However, contrary to what

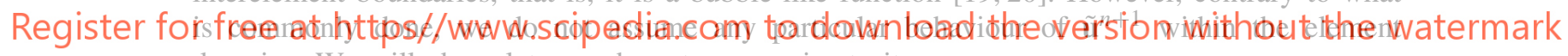
domains. We will show later on how to approximate it.

If in the time discrete problem $\mathbf{u}$ is replaced by $\mathbf{u}_{*}^{n+1}:=\mathbf{u}_{h}^{n+1}+\widetilde{\mathbf{u}}^{n+1}, p$ is replaced by $p_{h}^{n+1}$, the terms involving $\tilde{\mathbf{u}}^{n+1}$ are integrated by parts, and the test functions are taken in the finite element space, one gets

$$
\begin{aligned}
& \delta t \int_{\Omega}\left[v \nabla \mathbf{u}_{h}^{n+1}: \nabla \mathbf{v}_{h}+\left(\mathbf{u}_{h}^{n+1} \cdot \nabla \mathbf{u}_{h}^{n+1}\right) \cdot \mathbf{v}_{h}-p_{h}^{n+1} \nabla \cdot \mathbf{v}_{h}+q_{h} \nabla \cdot \mathbf{u}_{h}^{n+1}-\mathbf{f}^{n+1} \cdot \mathbf{v}_{h}\right] \mathrm{d} \Omega \\
& \quad+\int_{\Omega}\left[\mathbf{u}_{h}^{n+1}-\mathbf{u}_{h}^{n}\right] \cdot \mathbf{v}_{h} \mathrm{~d} \Omega-\delta t \int_{\Omega} \tilde{\mathbf{u}}^{n+1} \cdot\left(v \Delta_{h} \mathbf{v}_{h}+\mathbf{u}_{h}^{n+1} \cdot \nabla \mathbf{v}_{h}+\nabla q_{h}\right) \mathrm{d} \Omega=0
\end{aligned}
$$

where the notation $\Delta_{h}$ is used to indicate that the Laplacian needs to be evaluated element by element. Equation (17) must hold for all test functions $\mathbf{v}_{h}$ and $q_{h}$ in their corresponding finite element spaces.

The equation for the subscales $\tilde{\mathbf{u}}^{n+1}$ is obtained by taking the velocity test function in its space and $q=0$. The next step is to model the resulting equation. A possibility is to take [8,9]

$$
\tilde{\mathbf{u}}^{n+1}=\tau_{\mathrm{sgs}}\left[\mathbf{f}^{n+1}-\left(-v \Delta \mathbf{u}_{h}^{n+1}+\mathbf{u}_{h}^{n+1} \cdot \nabla \mathbf{u}_{h}^{n+1}+\nabla p_{h}^{n+1}\right)\right]=-\tau_{\mathrm{sgs}} \mathbf{R}_{h}^{n+1}
$$


where $\tau_{\text {sgs }}$ is a numerical parameter and $\mathbf{R}_{h}^{n+1}$ is the residual defined in (6) evaluated with $\mathbf{u}_{h}^{n+1}$. This is what we call algebraic approximation to the subscales. If, furthermore, we impose the subscales to be orthogonal to the finite element space, (18) has to be replaced by

$$
\tilde{\mathbf{u}}^{n+1}=-\tau_{\mathrm{sgs}} P_{h}^{\perp} \mathbf{R}_{h}^{n+1}=-\tau_{\mathrm{sgs}}\left(\mathbf{R}_{h}^{n+1}-P_{h}\left(\mathbf{R}_{h}^{n+1}\right)\right)
$$

where $P_{h}$ is the projection onto the finite element space. Since in this case, the subscales are orthogonal to the finite element space, we call this approach orthogonal subscales stabilization (OSS). The advantage of this approach is discussed in Reference [9]. From the accuracy point of view, it is less diffusive than the ASGS approach and yields better resolution of sharp gradients of the unknowns.

With all the approximations introduced heretofore, the final discrete problem to be solved for $\mathbf{u}_{h}^{n+1}$ and $p_{h}^{n+1}$ is

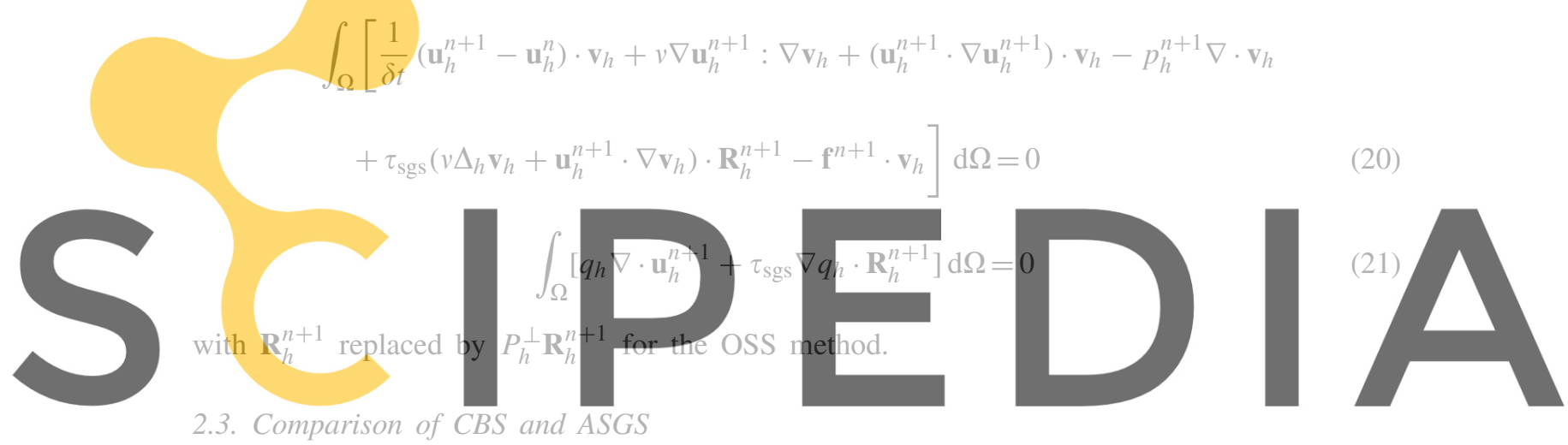

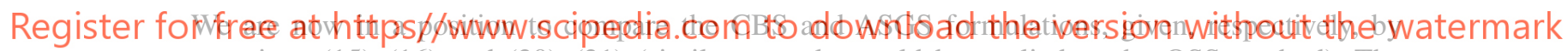
equations (15)-(16) and (20)-(21) (similar remarks could be applied to the OSS method). The differences between both methods are the following:

- The pressure gradient in the CBS method is extrapolated from values of the pressure in previous time steps. This does not affect the stabilization mechanism of the method, but only its implementation: it is possible to solve for the velocity first and to compute the pressure afterwards.

- The stabilization of the convective term is achieved in both cases through the introduction of streamline diffusion. The stabilizing terms are

$$
\begin{array}{ll}
\text { CBS: } & \tau_{\mathrm{cg}}\left(\hat{\mathbf{u}}_{h}^{n} \cdot \nabla \mathbf{v}_{h}\right) \cdot \hat{\mathbf{R}}_{h}^{n} \\
\text { ASGS: } & \tau_{\mathrm{sgs}}\left(v \Delta_{h} \mathbf{v}_{h}+\mathbf{u}_{h}^{n+1} \cdot \nabla \mathbf{v}_{h}\right) \cdot \mathbf{R}_{h}^{n+1}
\end{array}
$$

Except for the time level where the residual is evaluated (which is irrelevant for our discussion) and the viscous operator applied to the test function for the ASGS method (which is zero for linear elements) we see that both methods introduce the same amount of streamline diffusion if we identify $\tau_{\mathrm{sgs}}=\tau_{\mathrm{cg}}:=(2 \gamma-1) \delta t / 2$. Remember that $\gamma$ defines the position along the characteristics at which the equations are discretized in time. 
- Pressure is stabilized in both cases through the introduction of a pressure-Laplacian. For the CBS method, this is what is directly introduced, whereas for the ASGS formulation this term is part of the weighting of the element residual:

$$
\begin{array}{ll}
\text { CBS: } & \tau_{s} \nabla q_{h} \cdot \nabla p_{h}^{n+1} \\
\text { ASGS: } & \tau_{\mathrm{sgs}} \nabla q_{h} \cdot\left(-v \Delta \mathbf{u}_{h}^{n+1}+\mathbf{u}_{h}^{n+1} \cdot \nabla \mathbf{u}_{h}^{n+1}+\nabla p_{h}^{n+1}-\mathbf{f}^{n+1}\right)
\end{array}
$$

Remember that $\tau_{s}$ is given by (13) and it is not necessarily equal to $\tau_{\mathrm{cg}}$.

From this comparison we see that, even though the CBS and the ASGS methods start from different motivations, they have very similar stabilizing effects, both for convection and for pressure interpolation.

\section{NUMERICAL RESULTS}

In this section, we present the results of three classical numerical experiments we have performed using the stabilized formulations described in this paper. The goal is to compare the CBS method with the ASGS and OSS methods. The first two examples are steady-state cases,

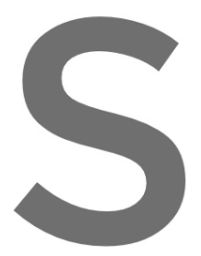
whereas the third is

3.1. Cavity flow

This benchmark test c cavity $\Omega=10,1[\times] 0,1$ when
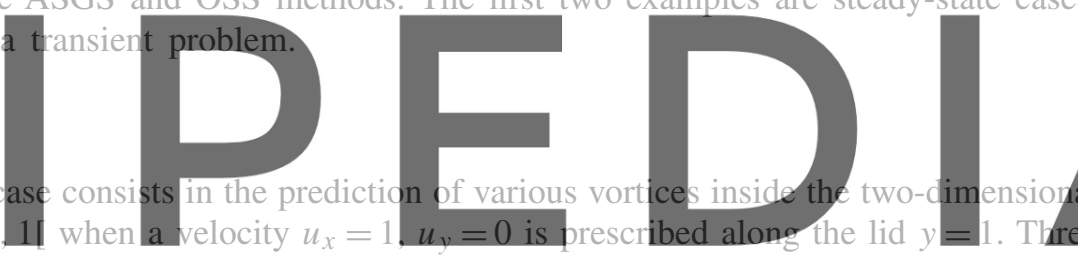

Reynolds numbers have been analysed: 400, 1000 and 5000.

triangular elements.

The general streamiine and pressure pattern for Reynolds number 5000 obtained with mesh unstructured 1 and the OSS method is shown in Figure 1.

To determine the accuracy of the numerical results we have compared them with those presented in Reference [21], which were obtained using a very fine grid and have become a standard reference. The comparison of the $x$ and $y$ velocity profiles along the cavity mid-sections $x=0.5$ and $y=0.5$ are shown in Figures 2-5. In all the cases, the time step has been chosen equal to the critical one determined by an explicit treatment of the viscous and convective terms (global time stepping is used). This is necessary for the CBS scheme, but used only for comparison in the case of SGS methods.

Table I. Meshes used for the cavity flow.

\begin{tabular}{lcr}
\hline Mesh & Npoin & Nelem \\
\hline Structured & 1521 & 2888 \\
Unstructured 1 & 5515 & 10596 \\
Unstructured 2 & 2929 & 5656 \\
\hline
\end{tabular}



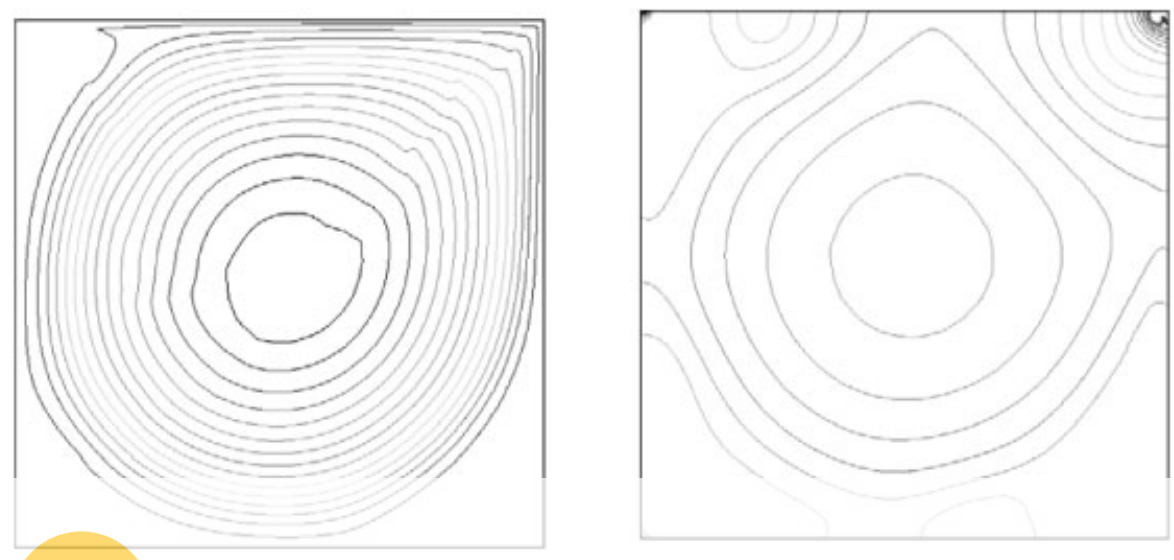

Figure 1. Streamline and pressure pattern for the cavity flow problem at Reynolds 5000 using the unstructured mesh 1 and the OSS method.
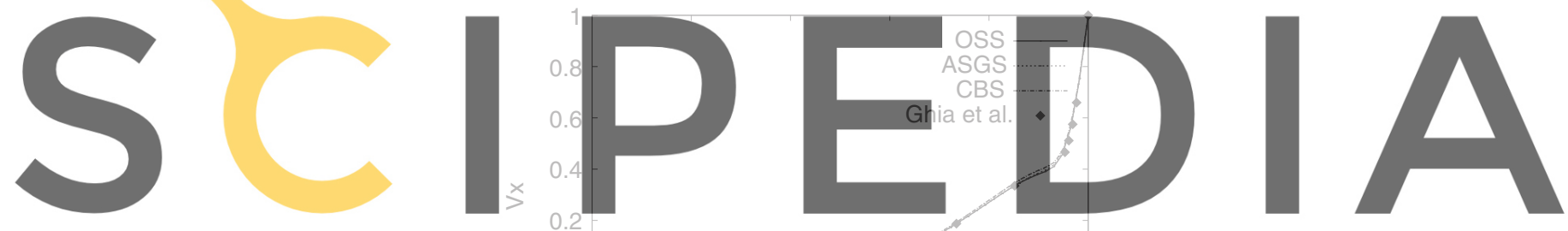

Register for free at https//www.scip̉edia.com to download the version without the watermark
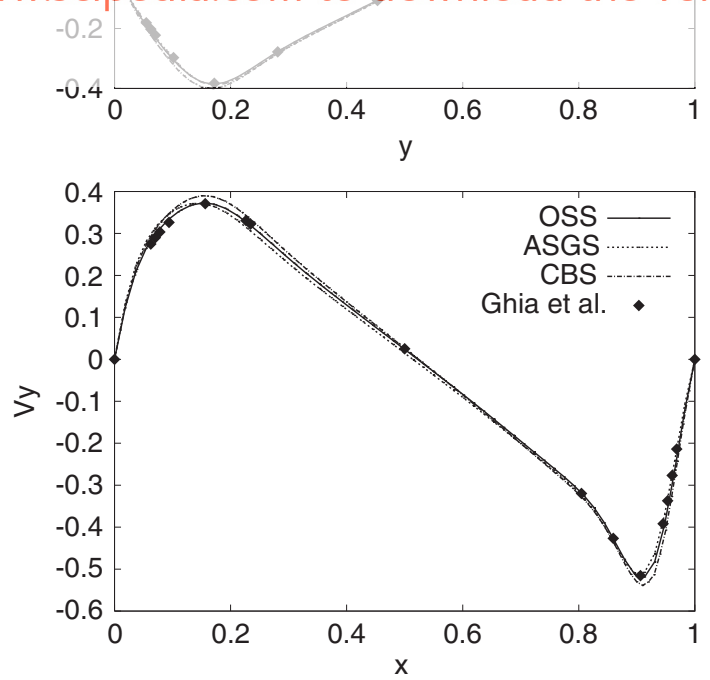

Figure 2. Velocity profiles along vertical and horizontal sections for a cavity flow at Reynolds 1000 using the unstructured mesh 2 . 

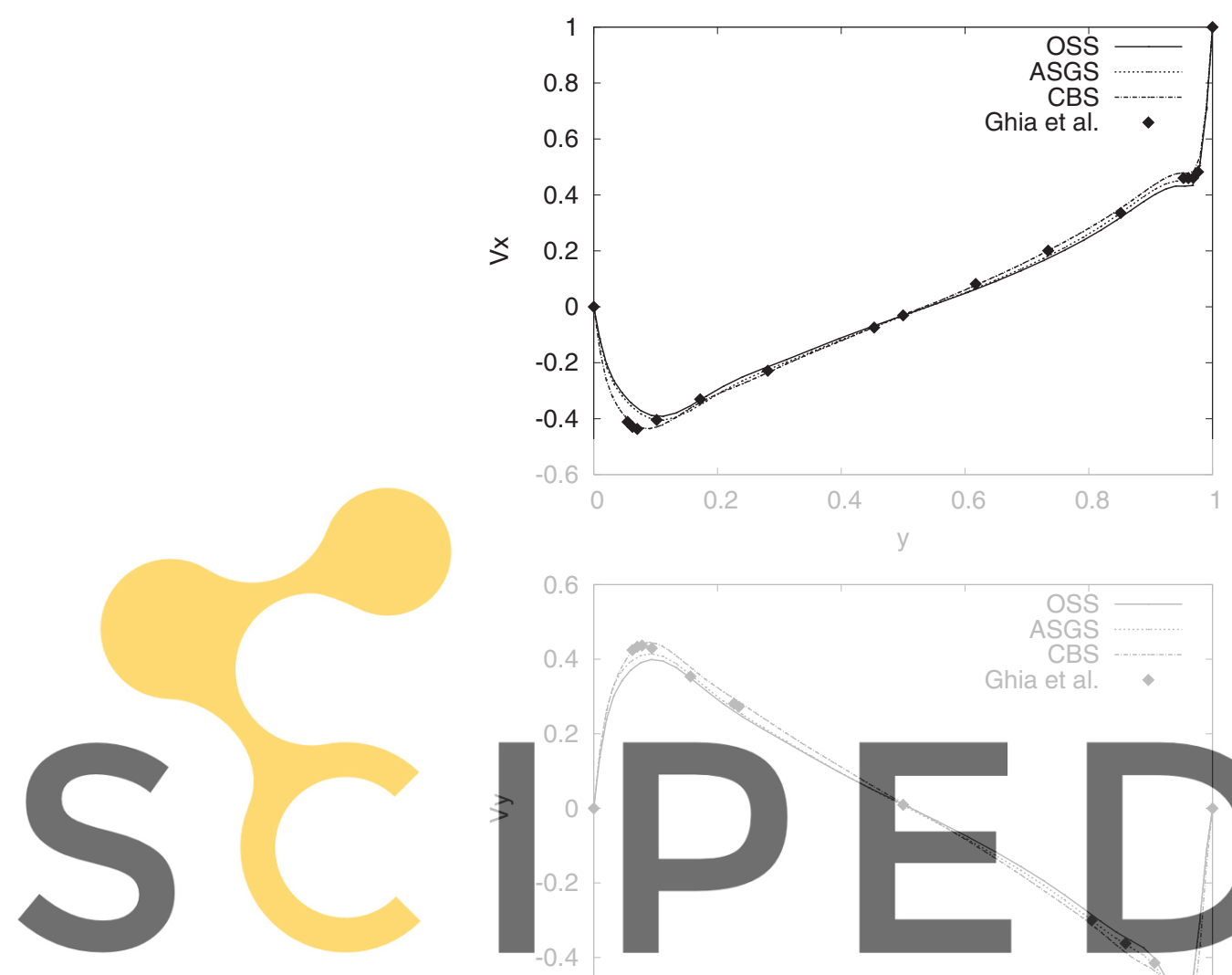

\section{Register for free at https//wwwọscipedia.com to download the version without the watermark}

Figure 3. Velocity profiles along vertical and horizontal sections for a cavity flow at Reynolds 5000 using the structured mesh.

It is observed from these figures that all methods lead to good results. The major differences are found for the case $R e=5000$ (results for $R e=400$ are not shown, since all methods give very similar answers). In the case of the structured mesh, the CBS scheme seems to perform better (results are closer to those of Reference [21]), whereas for the coarse unstructured mesh the best results are obtained with the OSS method. Thus, no conclusive comment can be made on which method performs best in this problem.

Another aspect that is numerically relevant is the way the solution evolves to the steady state. This gives an indication of the amount of numerical dissipation of the different methods. Figure 6 provides this comparison. It gives the norm of the difference in velocities from time step $n$ to $n+1$ normalized by the norm of the velocity at time step $n+1$. It is observed that ASGS and OSS display a very similar behaviour, whereas CBS is less monotone. This is possibly due to the implicit treatment of convection and viscosity in SGS methods and 

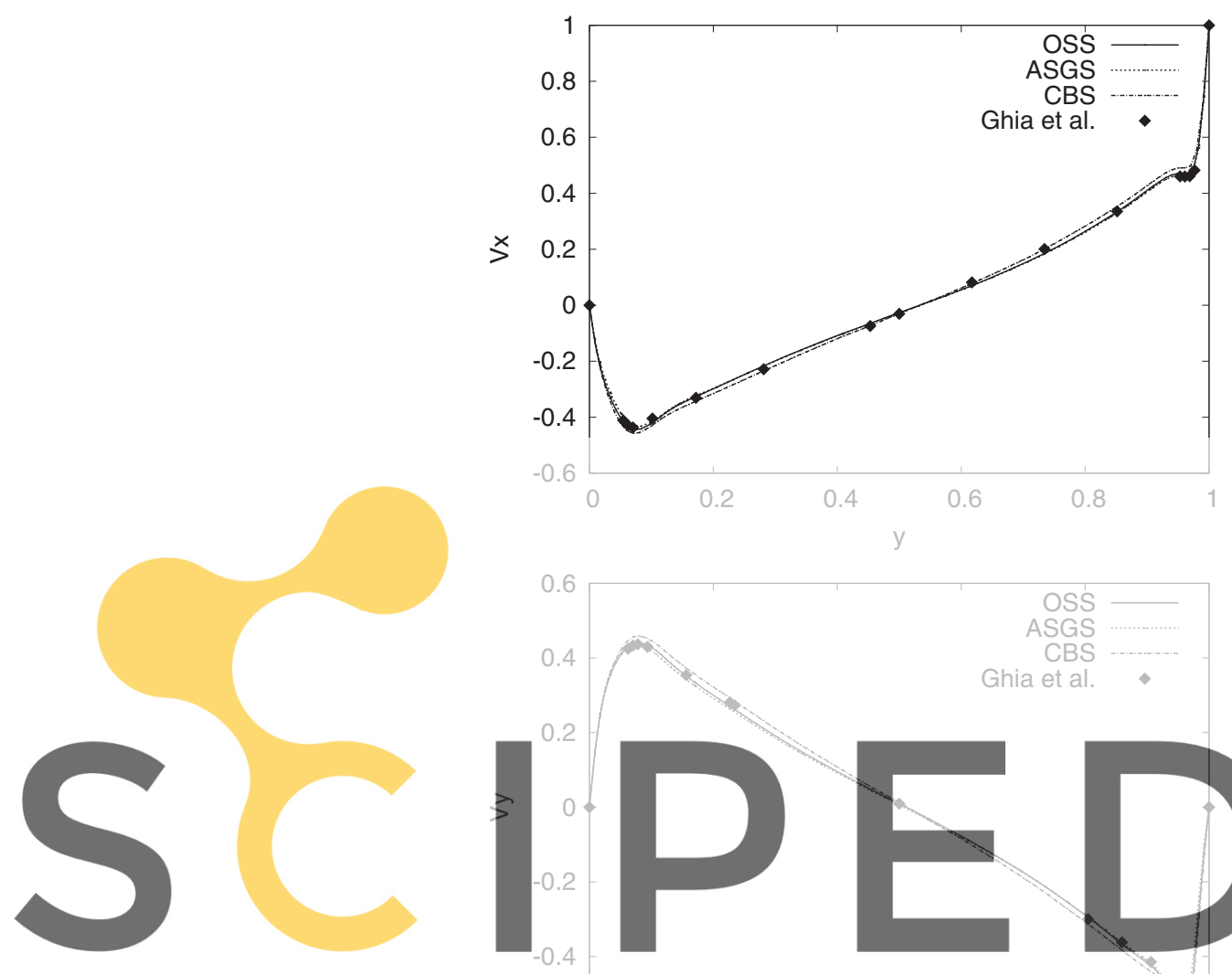

\section{Register for free at https//wwwosscipedia. com to to $_{0.2}$ download the version without the watermark}

Figure 4. Velocity profiles along vertical and horizontal sections for a cavity flow at Reynolds 5000 using the unstructured mesh 1.

the explicit one in CBS. For the coarse unstructured mesh, CBS fails to drop the residual below $10^{-3}$.

\subsection{Flow over a backward-facing step}

This second example is the classical benchmark of a flow over a backward-facing step. The length of the inflow channel is 4 and its width 2, the total length of the computational domain 40 and the width of the channel 3. The experimental parabolic velocity profile presented in Reference [22] is prescribed on the inflow, whereas the no-slip condition is prescribed on the rest of the walls except the outflow, where a zero traction condition is fixed. The Reynolds number, computed with a velocity 1 and the step height (1) is 229 .

The computational domain has been discretized using two unstructured meshes. The first one, referred to as mesh 1 in the following, consists of 8662 linear triangles and 4656 nodal points, 

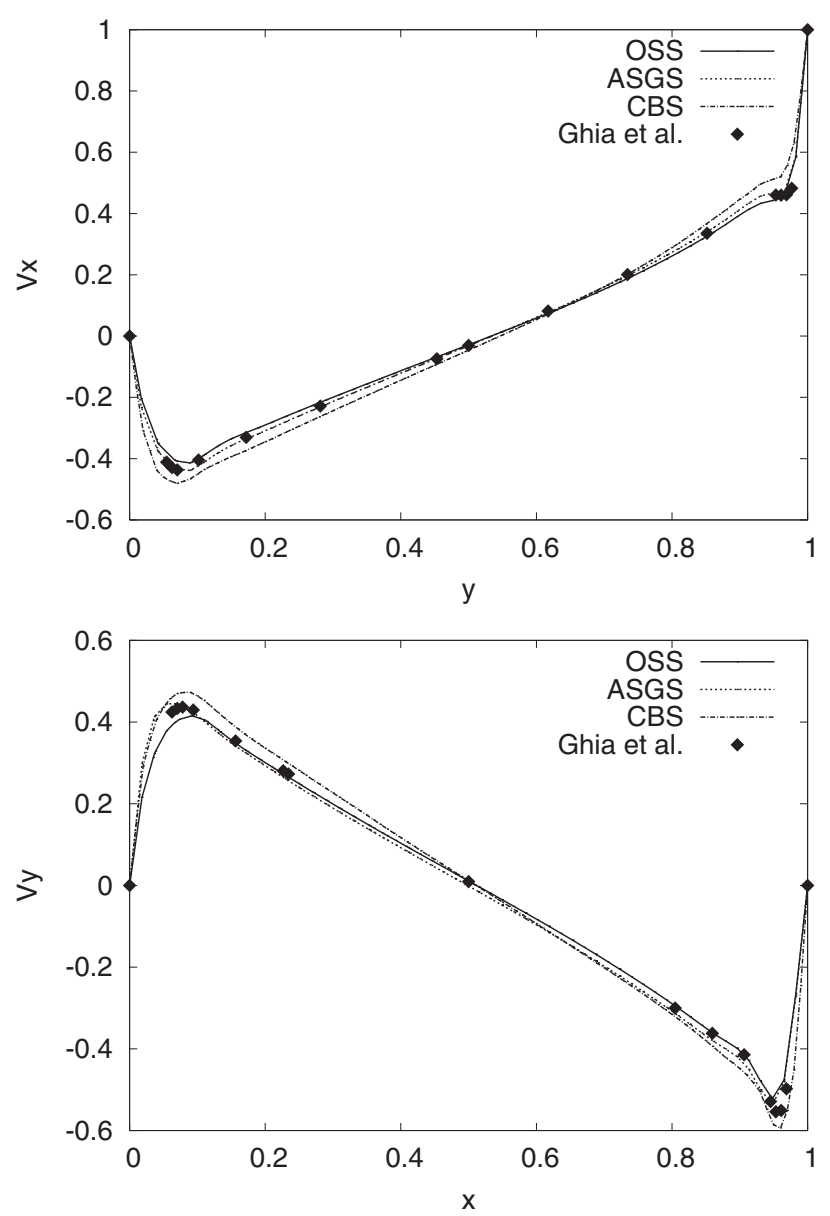

Figure 5. Velocity profiles along vertical and horizontal sections for a cavity flow at Reynolds 5000 using the unstructured mesh 2.

whereas the second, which we will call mesh 2, consists of 22257 linear triangles and 11659 nodal points. Both are refined near the boundaries. Again, the time step has been chosen equal to the critical one determined by an explicit treatment of the viscous and convective terms.

Figure 7 shows the pressure contours and the streamlines using mesh 2 and the OSS method. It is observed that in the region where the pressure is almost constant there are some localized pressure oscillations (for non-stabilized schemes these oscillations are global) which can be removed by increasing the stabilization parameter or computing it locally. We have preferred to take it constant all over the mesh and equal to one half of the critical time step to maintain the analogy between SGS schemes and CBS (which does not show these small local oscillations).

Figure 8 compares the horizontal velocity profiles along two vertical sections using mesh 1 with the experimental values obtained by Denham and Patrik [22]. For mesh 2 the results 

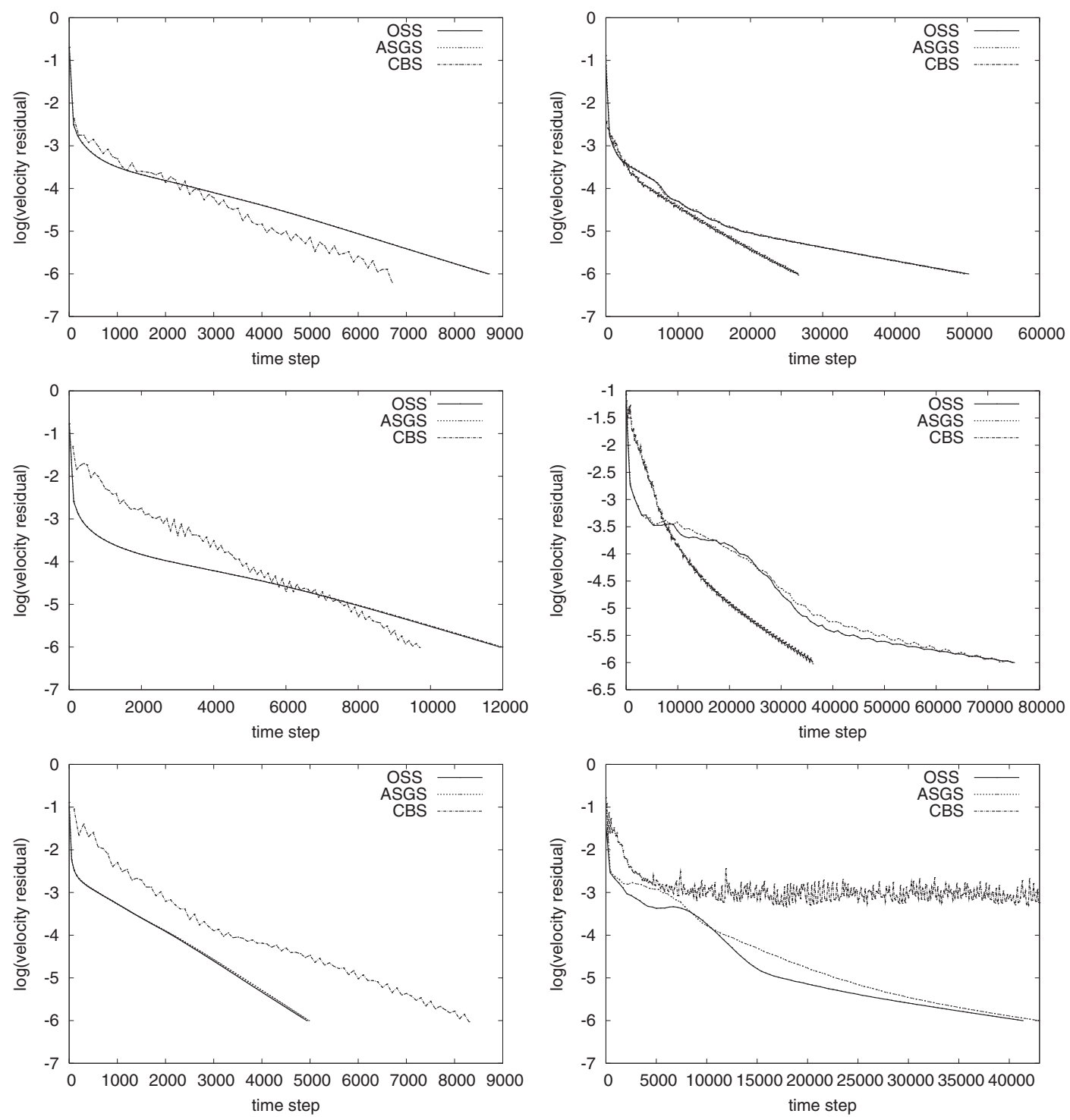

Figure 6. Convergence to the steady state for the flow inside a wall driven cavity at $R e=400$ (left) and $R e=5000$ (right) using the structured mesh (top), the unstructured mesh 1 (middle) and the unstructured mesh 2 (bottom).

are very similar to those obtained with mesh 1 and differences between the three methods are even smaller than those shown.

Figure 9 shows the convergence towards the steady state.

The conclusions from this example are the same as in the previous one, namely, the three methods considered yield very similar numerical answers. This confirms the similarity in the stabilization mechanisms identified for the three methods. 

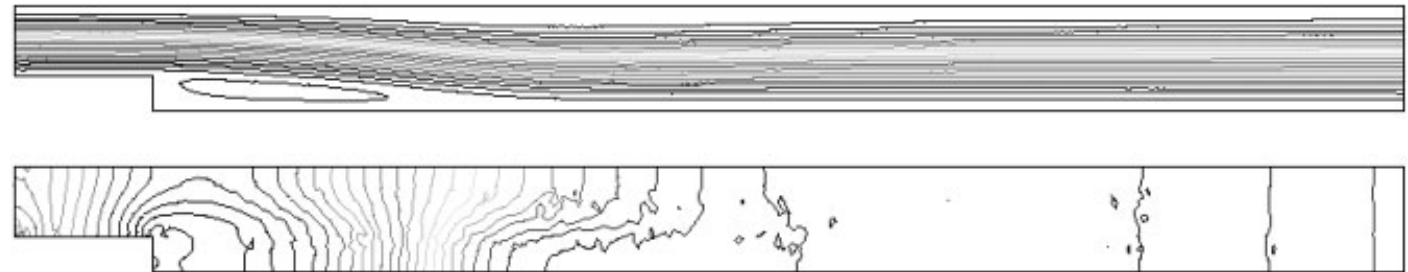

Figure 7. Streamline and pressure pattern for the BFS flow problem at Reynolds 229 using mesh 2 and the ASGS method.
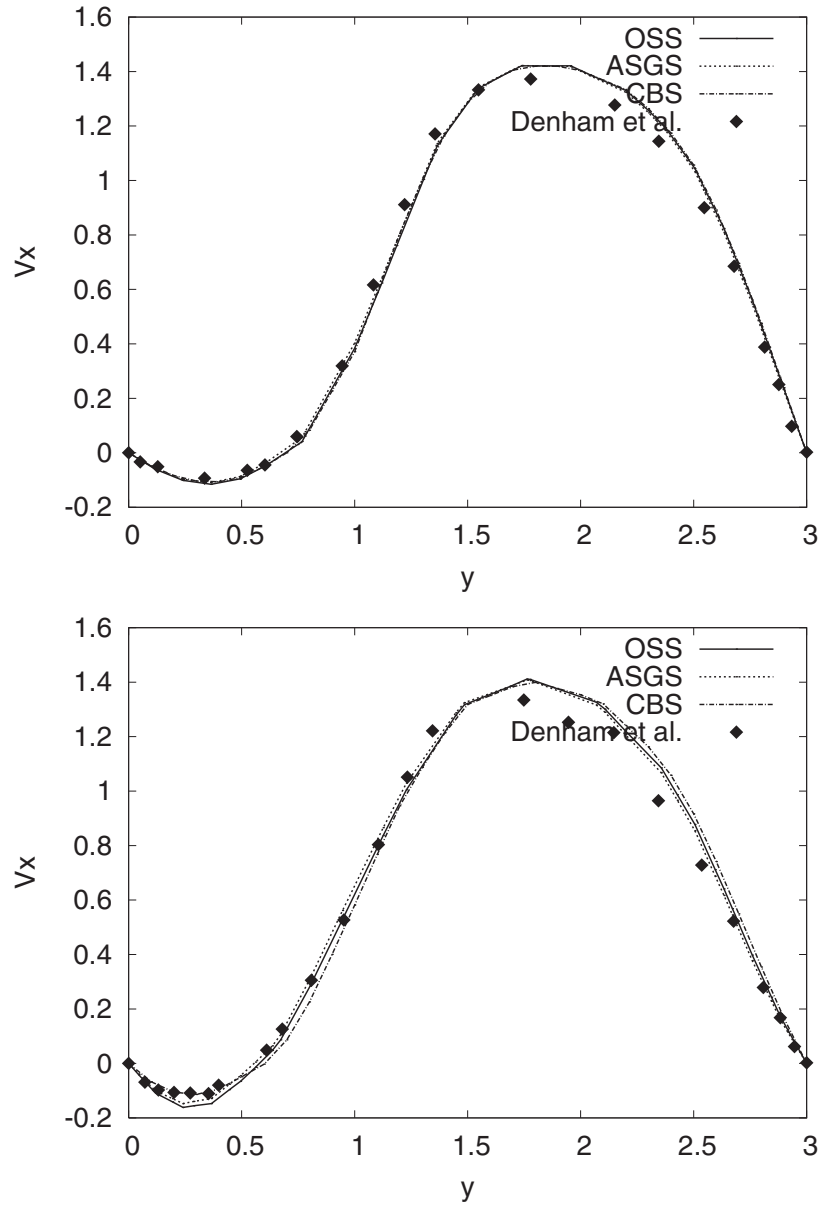

Figure 8. Horizontal velocity profiles along two vertical sections at $x=6.11$ (top) and 8.17 (bottom), for a BFS flow at $R e=229$ using mesh 1 . 

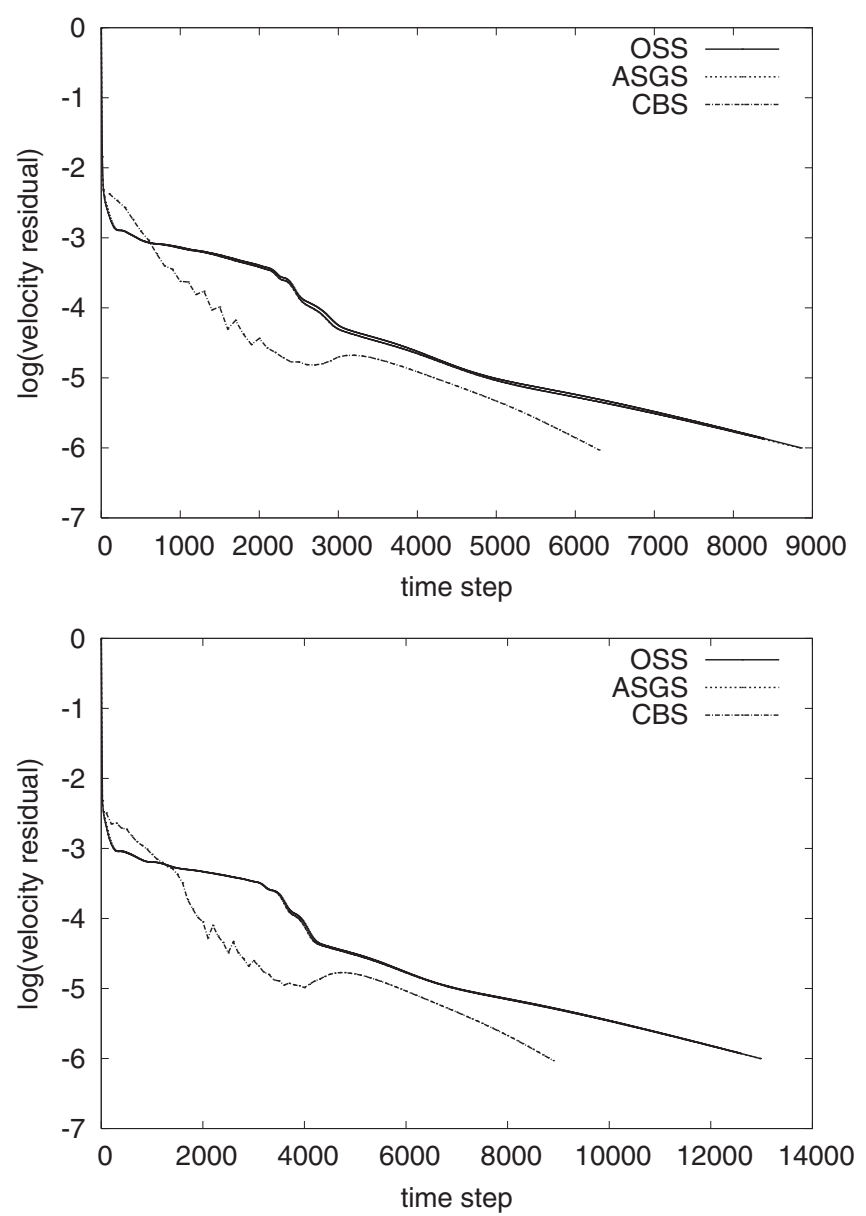

Figure 9. Convergence to the steady state for a BFS flow at $R e=229$ using mesh 1 (top) and mesh 2 (bottom).

\subsection{Flow over a cylinder}

This example involves the flow past a cylinder, another widely solved benchmark problem. The computational domain is $\bar{\Omega}=[0,16] \times[0,8] \backslash D$, with the cylinder $D$ of diameter 1 and centred at $(4,4)$. The velocity at $x=0$ is prescribed to $(1,0)$, whereas at $y=0$ and 8 , the $y$-velocity component is prescribed to 0 and the $x$ component is left free. The outflow (where both the $x$ and $y$ components are free) is $x=16$. The Reynolds number is 100 , based on the cylinder diameter and the prescribed inflow velocity. The finite element mesh employed consists of 19650 linear triangles, with 9988 nodal points, being refined near and behind the cylinder.

Figure 10 shows the streamlines and pressure pattern obtained using the OSS method.

The temporal evolution of the drag and lift coefficients, $C_{\mathrm{D}}$ and $C_{\mathrm{L}}$, as well as the evolution of the vertical velocity at the midpoint of the output section are shown in Figures 11 and 12 . The differences using the three methods in this example are more important than in the previous cases. The reason is due to that the CBS scheme employed is first-order accurate in 

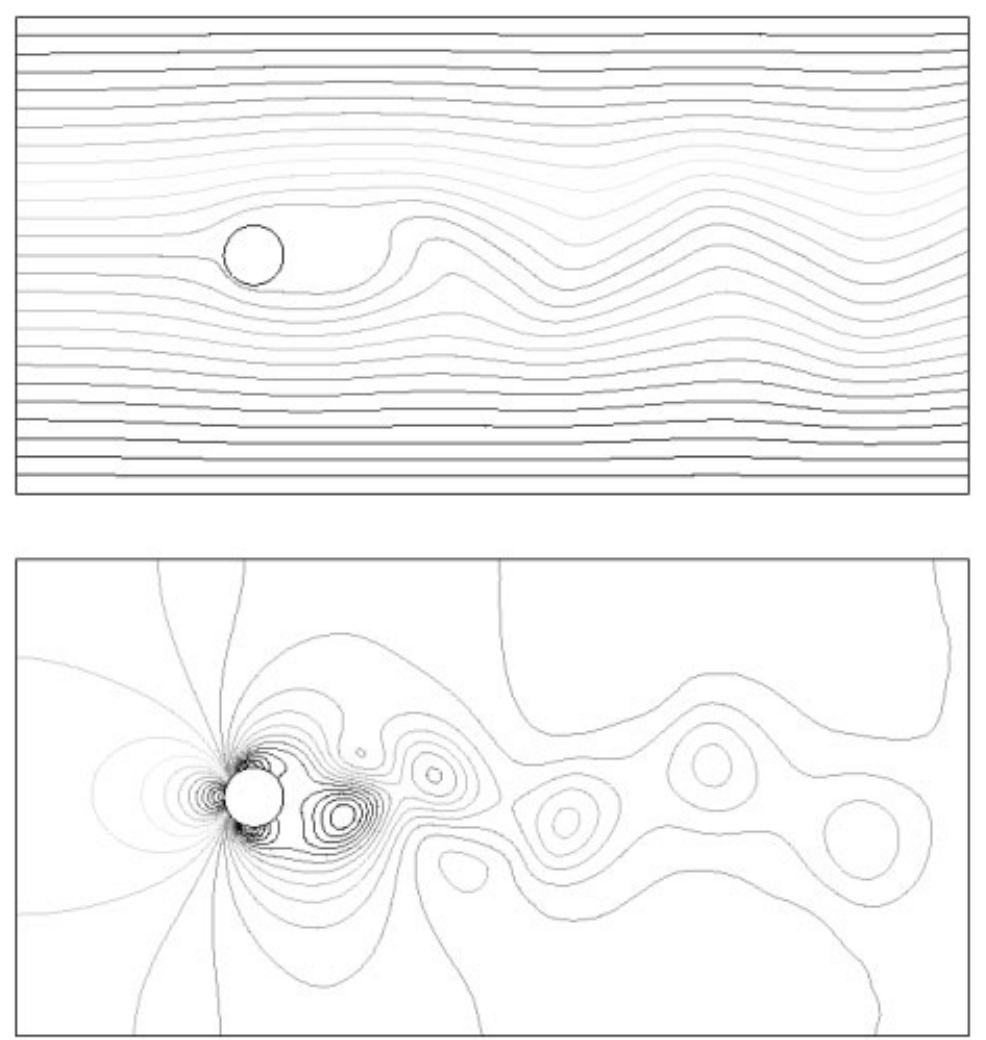

Figure 10. Streamline and pressure pattern for the flow over a cylinder at Reynolds 100 using the OSS method.

time (remember that for second-order splitting the stabilizing effect on the pressure disappears), whereas for OSS and ASGS, the second-order Crank-Nicolson scheme has been used. This leads to a more dissipative behaviour encountered for the CBS method, with smaller amplitude and frequency. Concerning the phase difference, it is due to very small frequency discrepancies (observe that the time window starts at $t=155$ ). Comparing ASGS and OSS, it is seen that the former is more dissipative. For a more detailed comparison of both methods, see Reference [9].

\subsection{CPU time comparison}

The analysis of the computational efficiency of the different schemes can be strongly biased depending on the criteria one uses to compare them. The main difficulty arises from the fact that OSS and ASGS are implicit and CBS is explicit.

For the implicit schemes, one can use a bigger time step than the critical one. But if this is done, the results presented in Figures 6 and 9 would have no sense. Therefore, despite the fact that OSS and ASGS are implicit, we forced them to run with a time step size equal to the critical one. The results are shown in Table II.

For the cylinder problem, the CBS method was used with a dual time stepping technique that enabled the time step size to be $0.1666 \mathrm{~s}$ (approximately 50 times the critical time step). 

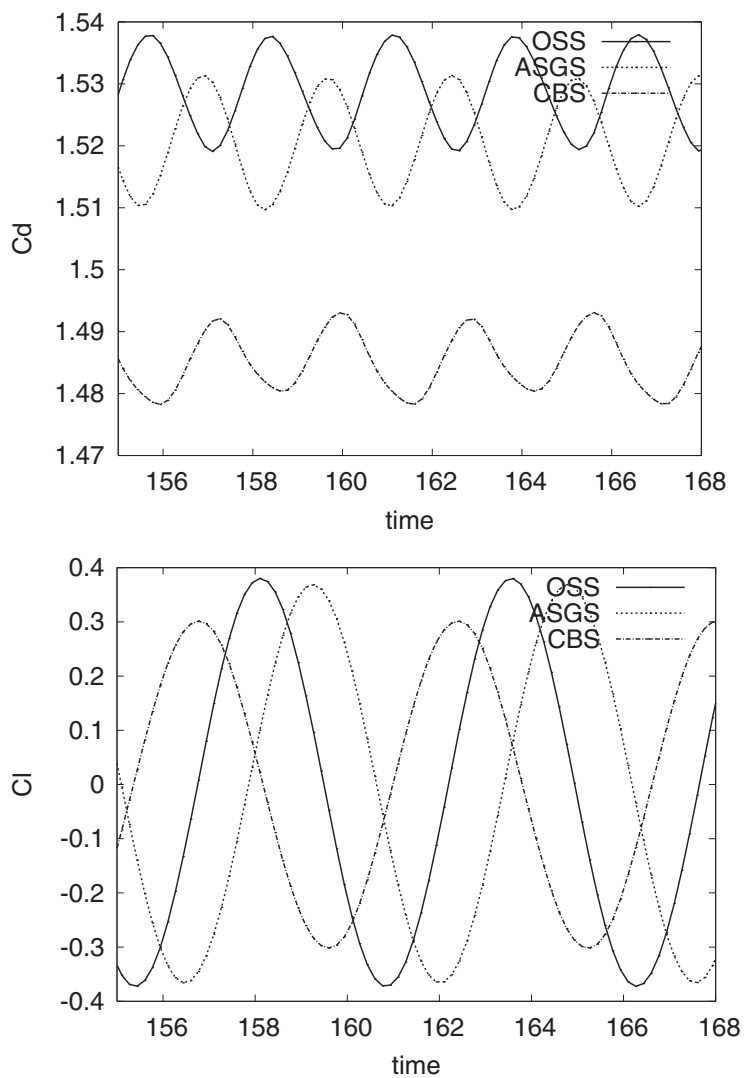

Figure 11. $C_{\mathrm{D}}$ and $C_{\mathrm{L}}$ for a flow around a cylinder at $R e=100$.

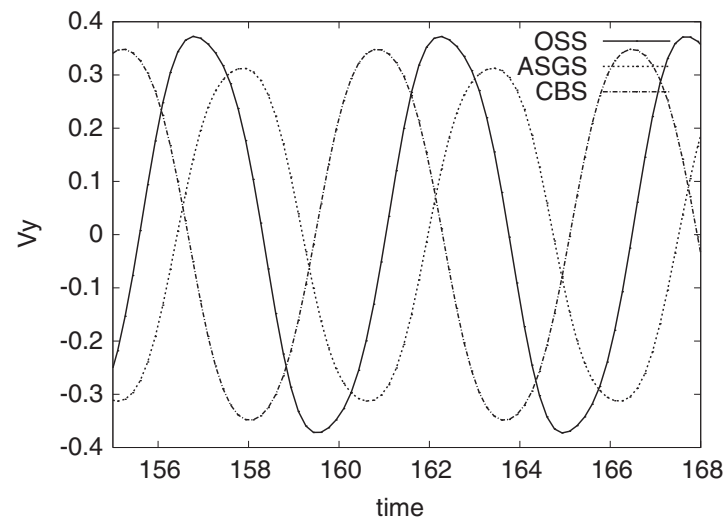

Figure 12. Vertical velocity for a flow around a cylinder at $R e=100$ at the midpoint of the output section. 
Table II. CPU time per time step normalized by the CPU time of an explicit update.

\begin{tabular}{lcrrrr}
\hline Mesh & CBS & ASGS $_{\text {mat }}$ & OSS $_{\text {mat }}$ & ASGS $_{\text {sol }}$ & OSS $_{\text {sol }}$ \\
\hline Cavity & & & & & \\
Struct. & 111.3 & 938.9 & 905.9 & 4561.3 & 4594.3 \\
Unstruct. 1 & 121.5 & 839.6 & 805.3 & 19993.8 & 20111.3 \\
Unstruct. 2 & 117.1 & 1094.0 & 1150.9 & 23656.1 & 23599.1 \\
Step & & & & & \\
Mesh 1 & 118.3 & 717.3 & 677.8 & 1657.8 & 1697.1 \\
Mesh 2 & 119.3 & 464.4 & 440.4 & 4184.2 & 4235.2 \\
Cylinder & $5.79 \mathrm{e} 5$ & $7.66 \mathrm{e} 2$ & $6.90 \mathrm{e} 2$ & $1.01 \mathrm{e} 4$ & $1.02 \mathrm{e} 4$ \\
\hline
\end{tabular}

The same time step size was used for the OSS and ASGS methods. The problem was run for 1201 steps that correspond to $200 \mathrm{~s}$.

In order to make the CPU times comparable although they were run on different computers they are non-dimensionalized dividing by a reference time. The reference time is obtained as the time needed to update the velocity in an explicit scheme once the RHS is known.

For the cavity and step problems, a direct solver was used for the ASGS and OSS methods. For the cylinder, the linear system was solved using a GMRES solver, allowing a maximum of 100 iterations with a tolerance of $10^{-8}$ and a Krylov dimension of 30 .

For the ASGS and the OSS methods, the total CPU time is the sum of the time invested to build up the matrix and right-hand side of the algebraic system and the time needed to solve it. Clearly, for the two first examples, cavity and step, the CBS method is much more efficient (recall though that much larger time steps could have been used for the implicit ASGS and OSS methods). However, the dual time-stepping technique used in the CBS method to allow time steps larger than the critical one is much less efficient than an iterative scheme for a truly implicit solver (although the CBS scheme could be made much faster by allowing certain diffusive effects). This is a general tendency that can be corrected either by using an implicit version of CBS (and using also an iterative scheme) or by using acceleration techniques in the dual time stepping. The analysis of both points is beyond the interest of this comparison.

\section{CONCLUSIONS}

In this paper, we have compared the CBS and two SGS formulations to solve the incompressible Navier-Stokes equations. The presentation has highlighted the stabilization mechanisms of these methods, showing that they are in fact very similar, both for the stabilization of the convective term and the pressure interpolation.

The numerical comparison yields no conclusive remarks, except that all methods perform very similarly. CBS is better in some cases, whereas SGS schemes perform better in others (in fact, OSS is in general more accurate than ASGS, see Reference [9]). Concerning the computer cost, for time steps of the order of the critical one it is certainly preferable to use CBS (or any other scheme with an explicit treatment of convection and viscous terms), but if the time step to be used is large, it seems better to use directly an implicit formulation. 


\section{REFERENCES}

1. Zienkiewicz OC, Taylor RL, Nithiarasu P. The Finite Element Method for Fluid Dynamics (6th edn). Butterworth-Heinemann: Stoneham, MA, 2006.

2. Codina R, Vázquez M, Zienkiewicz OC. A general algorithm for compressible and incompressible flow-Part III. The semi-implicit form. International Journal for Numerical Methods in Fluids 1998; 27:13-32.

3. Zienkiewicz OC, Codina R. A general algorithm for compressible and incompressible flow-Part I. The split, characteristic-based scheme. International Journal for Numerical Methods in Fluids 1995; 20:869-885.

4. Zienkiewicz OC, Nithiarasu P, Codina R, Vázquez M, Ortiz P. The characteristic based split procedure: an efficient and accurate algorithm for fluid problems. International Journal for Numerical Methods in Fluids 1999; 31:359-392.

5. Hughes TJR. Multiscale phenomena: Green's function, the Dirichlet-to-Neumann formulation, subgrid scale models, bubbles and the origins of stabilized formulations. Computer Methods in Applied Mechanics and Engineering 1995; 127:387-401.

6. Hughes TJR. Franca LP, Hulbert GM. A new finite element formulation for computational fluid dynamics: VIII. The Galerkin/least-squares method for advective-diffusive equations. Computer Methods in Applied Mechanics and Engineering 1989; 73:173-189.

7. Franca LP, Hughes TJR. Convergence analyses of Galerkin least-squares methods for advective-diffusive forms of the Stokes and incompressible Navier-Stokes equations. Computer Methods in Applied Mechanics and Engineering 1993; 105:285-298.

8. Codina R. A stabilized finite element method for generalized stationary incompressible flows. Computer Methods in Applied Mechanics and Engineering 2001; 190:2681-2706.

9. Codina R. Stabilized finite element approximation of transient incompressible flows using orthogonal subscales. Computer Methods in Applied Mechanics and Engineering 2002; 191:4295-4321.

10. Shakib F, Hughes TJR. A new finite element formulation for computational fluid dynamics: IX. Fourier analysis of space-time Galerkin/least-squares algorithms. Computer Methods in Applied Mechanics and Engineering 1991; 87:35-58.

11. Codina R, Zienkiewicz OC. CBS versus GLS stabilization of the incompressible Navier-Stokes equations and the role of the time step as stabilization parameter. Communications in Numerical Methods in Engineering 2002; 18:99-112.

12. Nithiarasu P. An efficient artificial compressibility (AC) scheme based on the characteristic based split (CBS) method for incompressible flows. International Journal for Numerical Methods in Engineering 2003; 56:1815-1845.

13. Nithiarasu P, Mathur JS, Weatherill NP, Morgan K. Three-dimensional incompressible flow calculations using the characteristic based split (CBS) scheme. International Journal for Numerical Methods in Fluids 2004; 44:1207-1229.

14. Chorin AJ. Numerical solution of the Navier-Stokes equations. Mathematics of Computation 1968; 22: 745-762.

15. Temam R. Sur l'approximation de la solution des équations de Navier-Stokes par la méthode des pas fractionaires (I). Archives for Rational Mechanics and Analysis 1969; 32:135-153.

16. Rannacher R. On Chorin's Projection Method for Incompressible Navier-Stokes Equations. Lecture Notes in Mathematics, vol. 1530. Springer: Berlin, 1992; 167-183.

17. Codina R. Pressure stability in fractional step finite element methods for incompressible flows. Journal of Computational Physics 2001; 170:112-140.

18. Brezzi F, Douglas J. Stabilized mixed methods for the Stokes problem. Numerische Mathematik 1988; 53:225-235.

19. Baiocchi C, Brezzi F, Franca LP. Virtual bubbles and Galerkin/least-squares type methods (Ga.L.S). Computer Methods in Applied Mechanics and Engineering 1993; 105:125-141.

20. Brezzi F, Franca LP, Hughes TJR, Russo A. $b=\int g$. Computer Methods in Applied Mechanics and Engineering 1997; 145:329-339.

21. Ghia U, Ghia KN, Shin CT. High-Re solutions for incompressible flow using the Navier-Stokes equations and a multi-grid method. Journal of Computational Physics 1982; 48:387-441.

22. Denham MK, Patrik MA. Laminar flow over a downstream-facing in a two-dimensional flow channel. Transactions of the Institution of Chemical Engineers 1974; 52:361-367. 